10.2478/gb-2021-0001

$\$$ sciendo

\title{
Durch fachliche Kontakte und unermessliche Wirkung höheren Ansprüchen dienend. Nachruf auf Prof. Dr. Horst Schuller ${ }^{1}$
}

Serving higher goals through professional contacts and immeasurable impact. In memoriam Prof.dr. Horst Schuller.

Am 25. Juli 2021 ist Horst Schuller, Literaturwissenschaftler, Literaturkritiker, Kulturvermittler, Übersetzer und Publizist, im Alter von knapp 81 Jahren verstorben. Der Lehrstuhl für Germanistik an der Lucian-Blaga-Universität in Hermannstadt verliert mit Horst Schuller ein Vorbild und einen treuen Weggefährten.

Horst Schuller kam 1990 an den neu gegründeten Lehrstuhl für Germanistik und hat hier vieles bewegt und erreicht. Zehn Jahre nach seiner Promotion an der Universität Bukarest wurde er 1994 zum ordentlichen Professor ernannt - und stärkte durch vernünftige Flexibilität fortan die Fachgemeinschaft und den Ruf des Lehrstuhls: Mit der Erforschung historisch-kultureller Zusammenhänge in multikulturellen und multiethnischen Räumen, der Entwicklung und Rezeption rumäniendeutscher Literatur und der rumänischen Literatur im deutschsprachigen Ausland erlangte der Lehrstuhl unter seiner Leitung ein zeitgemäßes wissenschaftliches Profil.

Horst Schuller, der in den 1990er-Jahren als Kulturreferent des Siebenbürgen-Forums aktiv war und den Fachbereich Germanistik laufend kritisch reflektierte, ermutigte die Umsetzung kultureller und wissenschaftlicher Initiativen oder trug, den organisatorischen Aufwand durchaus berücksichtigend, zur

1 Der Nachruf ist unter dem Titel Vorbild und treuer Weggefährte. Nachruf auf Prof. Dr. Horst Schuller (1940-2021) in der Hermannstädter Zeitung vom 6. August 2021, S. 4 erschienen. 
Wiederbelebung älterer, zuweilen in Vergessenheit geratener Projektideen bei. Sein Fachwissen im Spezialgebiet Neuere deutsche Literaturgeschichte, Komparatistik, Übersetzungswissenschaft und rumäniendeutsche Literatur brachte er vielfach und mit sachlicher Kritik ein. Sein Anliegen galt gesamtkulturellen und literaturhistorischen Entwicklungen im mehrsprachigen Kontext und der literarischen Rezeptions- und Wirkungsgeschichte. Daher förderte Horst Schuller jedwelche Form der kulturellen Betätigung und akademischer Einbringung und behielt dabei auch stets die Leistung in der Lehre oder Forschung im Auge.

Horst Schuller hat den Großteil der noch heute aktiven Lehrkräfte fachlich beraten und beruflich geformt. Bis zu seiner krankheitsbedingtenEmeritierung 2002 war Horst Schuller maßgeblich an der inhaltlichen Neuorientierung und Umgestaltung der Hermannstädter Germanistik beteiligt, deren Selbstverständnis unter neuartigen Bedingungen er durch wissenschaftliche Anregungen zur dokumentarischen Absicherung, durch komparatistische, imagologische oder interkulturelle Anstöße und kritisch-methodische sowie rezeptionsästhetische Auseinandersetzungen mit Literatur, Sprache und Kultur vorbildlich zu prägen verstand.

Der am 13. August 1940 in Meschen/Moșna bei Mediasch geborene Horst Schuller arbeitete nach Abschluss des Studiums der Germanistik und Rumänistik zuerst sechs Jahre lang als Deutschlehrer in Marienburg/Feldioara im Burzenland.

Danach übernahm er 1968 als Redakteur das Kulturressort der neu gegründeten Kronstädter Zeitung Karpatenrundschau. Auch leitete er den Literaturkreis der Karpatenrundschau und veranstaltete regelmäßig in verschiedenen Ortschaften stattfindende Mundartautorentreffen und Diskussionen mit öffentlichen Lesungen. Sachkundig und engagiert betreute Horst Schuller jahrelang die Mundartrubrik „Vill Sprochen än der Wält" und organisierte Mundartdichtertreffen. Ehrenamtlich 
wirkte er jahrelang als Mitglied der Kulturkommission des Siebenbürgen-Forums mit.

Nachdem Horst Schuller im Jahr 2000 die Leitung des Lehrstuhls übernahm, erfuhr die Hermannstädter Germanistik einen Aufschwung. Bisweilen überraschende administrative Wirrungen und Verwicklungen fanden durch seine Erfahrung und Menschlichkeit eine glückliche Fügung. Zahlreiche wissenschaftliche Kooperationen und Partnerschaften wurden von ihm angeregt und umgesetzt. Erinnert sei hier bloß an die überaus erfolgreiche Partnerschaft mit der Philipps-Universität Marburg und die Kooperation mit dem Internationalen Zentrum für Wissenschaftliche Zusammenarbeit der Eberhard-Karls-Universität Tübingen. Das Organisationstalent von Horst Schuller kam zudem bei zahlreichen Veranstaltungen zur Geltung. Die im August 1997 innerhalb einer Sommerakademie abgehaltene Tagung, ,Germanistik im internationalen Vergleich. Zur Reform des Faches in Mittel- und Südosteuropa" (vgl. Germanistische Beiträge 8-9/1998, S. 7-13) mit Gästen aus zehn Ländern oder die Gedenkfahrt zu den Lebensstätten von Stephan Ludwig Roth (1796-1849) im Mai 1996 gehören zu den Ereignissen, die das Ansehen der Hermannstädter Germanistik zweifellos gestärkt haben.

Als Lehrstuhlinhaberund Mitherausgeber derFachzeitschrift Germanistische Beiträge hat Professor Schuller stets motivierte, nicht minder kompetente Germanistinnen und Germanisten, seien es erfahrene Wissenschaftler oder Nachwuchsforscher, gefördert. Eine ganze Generation von Doktorandinnen und Doktoranden, landesweit dürften es fast 30 an der Zahl sein, haben ihn als geduldigen, gründlichen und anspruchsvollen Doktorvater erlebt. Nach seiner Ausreise 2003 blieb er als beratender Professor und Doktorvater im Fachbereich vergleichende Literaturwissenschaft, Übersetzungskritik, Rezeptionsgeschichte dem Lehrstuhl weiterhin verbunden und spendete einen beachtlichen Teil seiner Privatbibliothek dem Lehrstuhl. 
Nachdem an der Hermannstädter Germanistik 2008 das Zentrum für rumäniendeutsche Literatur eingerichtet worden war, konnte dieser Buchbestand von den hiesigen Germanisten, Nachwuchswissenschaftlern und Doktoranden zu Dokumentationszwecken genutzt werden.

Gekonnt verstand Horst Schuller es, richtungsweisend zu wirken, ein vorzügliches Arbeitsklima zu schaffen und kollegiale Verbundenheit zu fördern. Rücken wir ihn näher ins rechte Licht: Ein von Sachlichkeit und Höflichkeit bestimmter Umgang mit den Mitmenschen, Interesse für die Erkundung der Vielfalt des Miteinanders unterschiedlicher literarhistorischer, kultureller und sprachlicher Traditionen, Personen- und Sachkenntnis für die gemeinsame Sache einsetzend. In diesem Sinne agierte Horst Schuller. Man arbeitete gern mit ihm zusammen und man konnte offen miteinander umgehen. So haben wir ihn gekannt und so möchten wir ihn in Erinnerung bewahren: warmherzig und vornehm, gewissenhaft und wohlwollend, engagiert und taktvoll - damit auch weit über seinen Wirkungsort geschätzt und respektiert.

Wir nehmen nun Abschied von einem besonderen Menschen und verdienstvollen Fachkollegen. Den Beteiligten an Zusammenkünften, Kulturabenden, Mundartautorentreffen, Rundtischgesprächen, Lesungen und Tagungen im In- und Ausland wird er wohl als ein moderater Moderator im Gedächtnis bleiben: Verbindendes aufzeigend, über Trennendes vernünftig und taktvoll hinwegsehend. Horst Schuller war nicht nur ein weitsichtiger Lehrstuhlinhaber, kompetenter Literaturwissenschaftler und geachteter Hochschullehrer, sondern vor allem ein geduldiger Zuhörer mit Sinn für den feinen Humor.

Der vielseitig wirkende Germanist Horst Schuller, der in Hermannstadt ein wissenschaftliches Zuhause fand, war als Publizist und Forscher äußerst produktiv und erfolgreich. Seine Dissertation, Kontakt und Wirkung. Literarische Tendenzen in der siebenbürgischen Kulturzeitschrift ,Klingsor “, die erst 1994 
im Bukarester Kriterion-Verlag erscheinen konnte, zeigt Kontaktbeziehungen zwischen der rumäniendeutschen und rumänischen Literatur auf, wobei sich der Autor der siebenbürgischen Zeitschrift Klingsor (1924-1939) und ihrer kulturpolitischen und literarischen Vermittlerrolle widmete. Die Arbeit beleuchtet somit Entwicklungen der rumäniendeutschen Literatur und Querverbindungen $\mathrm{zu}$ weiteren rumänischen und ungarischen Publikationen in der Zwischenkriegszeit.

Horst Schuller veröffentlichte zahlreiche akribisch dokumentierte und wissenschaftlich solide literatur- und kulturhistorische Studien, Studienbände und Ausgaben. Mit seinem Namen und Wirken sind umfassende historisch-thematische Darstellungen zur rumäniendeutschen Literatur- und Kulturlandschaft, Essays zum deutschsprachigen Schulwesen und zur Lage der deutschen Minderheit in Rumänien sowie Beiträge verbunden, die sich mit Fragen der Rezeption rumänischer, rumäniendeutscher oder ungarischer Autorinnen und Autoren auseinandersetzen. Eine beträchtliche Anzahl an Publikationen zu einschlägigen Themen seines Lehr- und Forschungsgebiets und Rezensionen sind in den Fachzeitschriften germanistischer Standorte, in Jahrbüchern oder Sammelbänden erschienen. Als Projektleiter, Herausgeber von Editionen (Michael Königes, Friedrich Wilhelm Schuster, Georg Maurer, Frida Binder, Otto Fritz Jickeli, Johann Karl Schuller, Walter Peter Plajer) und Anthologien hat sich Horst Schuller nicht ausschließlich um die Vermittlung der rumäniendeutschen Literatur und Mundartdichtung verdient gemacht, sondern auch um ein besseres Verständnis des Literatur- und Kulturtransfers und der vielfältigen Aufgaben und Leistungen bemüht, die Vermittlungsinstanzen (Presseorgane, Redakteure, Schriftsteller) und Vertreter der siebenbürgisch-sächsischen Kultur für die Bewahrung der kollektiven Kohäsion und Identität beanspruchen können.

Als geschätzter Mitarbeiter diverser Publikationen räumte Horst Schuller transkulturellen Aspekten im rumänisch- 
deutschen Übersetzungsprozess einen gebührenden Platz ein und veröffentlichte in namhaften Periodika wie Karpatenrundschau oder Neue Literatur rezeptionsgeschichtliche Studien zur Lyrik und Prosa von Marin Sorescu, Ana Blandiana, Liviu Rebreanu oder Mihail Sadoveanu im deutschen Sprachraum, übersetzungskritische Arbeiten (Ludwig Vinzenz Fischer, Zoltan Franyó, Alfred Margul-Sperber). Auch würdigte er Übersetzungsleistungen und ging der Geschichte der deutschen Literaturübersetzungen aus dem Rumänischen nach.

Nach seiner Übersiedlung widmete er sich vornehmlich der Ergänzung lückenhafter Quellenverzeichnisse, verfolgte jedoch die Entwicklungen in der rumänischen Germanistik und beteiligte sich weiterhin an Diskussionsrunden und Tagungen. In den letzten Jahren hat Horst Schuller zahlreiche Quellen gesichtet und sich der sachlich-kritischen Darstellung der Vermittlungstätigkeit von bekannten und weniger bekannten Übersetzerinnen und Übersetzern zugewandt und sich damit den Bemühungen zur Bekanntmachung und Verbreitung der rumänischen Literatur angeschlossen. Es handelt sich hierbei um ein ehemaliges Großprojekt der Hermannstädter Germanistik, das Mitte der 1990er-Jahre von Lehrkräften und Studierenden in Angriff genommen und aus Mitteln der Volkswagenstiftung getragen wurde. Horst Schuller hat nach seiner Emeritierung die Forschungsarbeit hierzu nicht unterbrochen. Mit Beharrlichkeit hat er Archive und Nachlässe gesichtet und ab 2013 in einer Aufsatzreihe Porträts literarischer Übersetzerinnen und Übersetzer mehrerer Generationen aus ausgewählten Regionen Siebenbürgens zusammengestellt, die einen Einblick in die Übersetzungsproduktion rumänischer literarischer Zeugnisse ins Deutsche bieten. Da das Wirken dieser Kulturvermittler der breiteren Öffentlichkeit noch nicht vollumfänglich zugänglich gemacht wurde - Informationen zu den übersetzten Werken liegen nur vereinzelt und daher verstreut in übersetzungsgeschichtlichen oder literaturkritischen Beiträgen oder in kaum 
noch zugänglichen Buchpublikationen und Pressezeugnissen vor und werden daher oft ignoriert -, hat Horst Schuller die Produktion und Resonanz der Übersetzungsarbeit durch Auszeichnungen, Rezensionen oder kritische Stimmen gründlich dokumentiert und dadurch für weitere Untersuchungen im Bereich der (interkulturellen) Germanistik und Romanistik, für Fragen der Translations-, Sprach- und Kulturwissenschaft erschlossen.

Horst Schuller hat die Hermannstädter Germanistik über Jahre hindurch in akademischen und wissenschaftlichen Gremien, in internationalen Gesellschaften und Verbänden vertreten. Sein Wirken in Redaktionskollegien im In- und Ausland, die Beteiligung an mehreren Forschungsprojekten (Internationales Germanistenlexikon 1998 und Österreichisches Biografisches Lexikon 1998), die wissenschaftliche Zusammenarbeit mit Vertretern germanistischer Institutionen und Stiftungen europaweit und die zahlreichen Gastvorträge in Belgien, Dänemark, Deutschland, Österreich, Polen, Slowenien oder Ungarn haben zur besseren Wahrnehmung der hiesigen Germanistik beigetragen und ihren guten Ruf gestärkt. Unser Lehrstuhl würdigte sein vielfaches Engagement und widmete ihm zu runden Geburtstagen die Hefte 13/14 (2000) und 27 (2010) der Germanistischen Beiträge, die er jahrelang betreute. Ihm zu Ehren wurde auch im November 2010, anlässlich seines 70. Geburtstages, in Kooperation mit dem Institut für deutsche Kultur und Geschichte Südosteuropas an der Ludwig-Maximilians-Universität München (IKGS) die traditionelle Jahresagung ausgerichtet, die auf das multikulturelle Siebenbürgen fokussierte und interkulturelle Bezüge aufzudecken bemüht war, womit thematisch der Kern seines wissenschaftlichen Interesses umrissen wurde.

Für sein vielfältiges wissenschaftliches und kulturelles Wirken wurde Prof. Dr. Horst Schuller mit Auszeichnungen und Preisen geehrt. Er war Preisträger des Rumänischen Schriftstellerverbandes (1994 und 2000). 2018 erhielt er von den 
Verbänden der Siebenbürger Sachsen in Deutschland und Österreich den Siebenbürgisch-sächsischen Kulturpreis, womit beides - seine hervorragenden Kontakte und fortwährende Wirkung - und damit sein Tagewerk zur identitätsfördernden Aufbauarbeit Anerkennung fand und auch ein von Würde, Umsicht und Weisheit gelenktes Wirken, das künftige Herausforderungen überdauern wird.

Professor Dr. Horst Schuller war nicht der Größte, allerdings der Beste.

Einer der edlen Sorte.

Ruhe sanft, lieber Herr Professor.

Doris Sava 